\section{Glaucoma screening by optometrists: positive predictive value of visual field testing}

\begin{abstract}
Purpose Visual field testing is increasingly being performed by optometrists in order to improve glaucoma detection. The aim of this study was to assess the positive predictive value (PPV) of visual field testing as currently practised by optometrists.

Methods A retrospective study was performed of referrals for suspected glaucoma to an ophthalmology department during a 3 month period. The PPV for glaucoma was determined according to the reason for referral.

Results There were 86 referrals for suspected glaucoma. Isolated field loss accounted for $12 \%$ of these referrals but no cases of glaucoma detection. These subjects were either false positive referrals or had field defects of nonglaucomatous aetiology (tilted optic disc and porencephalic cyst). The PPV for glaucoma among referrals with isolated field loss was significantly less than that among referrals with field loss in association with corroborative abnormalities ( 0 vs $60 \%$,

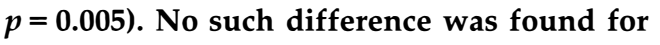
referrals with raised intraocular pressure $(43 \%$ vs $57 \%, p=0.38)$ or suspicious discs $(25 \%$ vs $53 \%, p=0.60)$.

Conclusions Visual field testing is currently leading to unnecessary referrals to the hospital eye service with no detectable improvement in the accuracy of glaucoma suspect referrals. It is important that optometrists perform visual field testing in accordance with validated screening methodology.
\end{abstract}

Key words Glaucoma, Mass screening, Ophthalmoscopy, Perimetry, Predictive value of tests, Tonometry

Glaucoma screening in the United Kingdom is mainly performed by optometrists through opportunistic screening of individuals attending for eyesight tests. ${ }^{1-3}$ Tonometry and ophthalmoscopy by optometrists are wellestablished screening tests for glaucoma. ${ }^{4}$ More recently, perimetry has become feasible as a screening test following the development of computerised perimeters that allow rapid, semi-
DOUGLAS K. NEWMAN, SAMIRA ANWAR, KERRY JORDAN

automated visual field testing. ${ }^{5}$ Visual field testing by optometrists is advocated both to improve the accuracy of referrals for suspected glaucoma and to increase the overall glaucoma detection rate. ${ }^{6}$

Perimetry currently achieves acceptable validity as a screening test for glaucoma only under certain conditions. These include the use of a perimeter that has satisfactory sensitivity and specificity for detecting glaucomatous field loss, ${ }^{7,8}$ selective screening of a population at increased risk of glaucoma, ${ }^{6,9}$ and repeating abnormal perimetry (in the absence of other features of glaucoma) to confirm genuine field loss before referral. ${ }^{6,10}$ Visual field testing performed without due regard to validated screening methodology has the potential to greatly increase false positive referrals from glaucoma screening without significantly improving the overall glaucoma detection rate. This study was performed to assess the positive predictive value (PPV) of visual field testing as currently practised by optometrists.

\section{Materials and methods}

A retrospective study was performed of referrals for suspected glaucoma to the department of ophthalmology at the West Suffolk Hospital during the 3 month period May to July 1994. These referrals were identified by reviewing the medical records of all patients referred to the department during this period (excluding emergency referrals). The West Suffolk Hospital has a catchment population of approximately 250000 . The medical records of those patients referred for suspected glaucoma were then reviewed to determine the reason for referral and the subsequent hospital eye service (HES) findings.

For the purposes of this study, the following definitions were applied to the HES findings. Glaucoma was defined as intraocular pressure (IOP) $>21 \mathrm{mmHg}$ together with characteristic optic disc and/or visual field changes, as judged by the examining ophthalmologist. Normal tension glaucoma was defined as the presence of characteristic optic disc and/or visual field changes with IOP consistently

\author{
D.K. Newman \\ S. Anwar \\ K. Jordan \\ Department of \\ Ophthalmology \\ West Suffolk Hospital \\ Bury St Edmunds \\ Suffolk, UK \\ D.K. Newman, MA, \\ FRCOphth \\ Department of \\ Ophthalmology \\ Clinic 3 (Box 41) \\ Addenbrooke's Hospital \\ Hills Road \\ Cambridge CB2 2QQ, UK \\ Tel: +44 (0)1223245151 \\ Fax: +44 (0)1223 217968
}


Table 1. Hospital eye service (HES) findings in referrals for suspected glaucoma

\begin{tabular}{lc}
\hline HES findings & No. of patients \\
\hline Glaucoma $^{\mathrm{a}}$ & 33 \\
No abnormality $_{\text {Ocular hypertension }^{\mathrm{b}}}$ & 24 \\
Normal tension glaucoma & 16 \\
Pigment dispersion syndrome $^{\mathrm{c}}$ & 2 \\
Other disorders $^{\mathrm{c}}$ & 1 \\
Unknown & 6 \\
\hline
\end{tabular}

${ }^{a}$ The types of glaucoma diagnosed were primary open-angle glaucoma (28), pseudoexfoliative glaucoma (3), aphakic glaucoma (1) and chronic angle-closure glaucoma (1). ${ }^{b}$ Seven patients with ocular hypertension had an IOP $\geqslant 28 \mathrm{mmHg}$. 'Other disorders were tilted optic disc (4), porencephalic cyst (1) and myopic macular degeneration (1).

$\leqslant 21 \mathrm{mmHg}$. Ocular hypertension was defined as IOP $>21 \mathrm{mmHg}$ with no optic disc or visual field abnormalities. The examining ophthalmologists at the HES used Goldmann applanation tonometry, stereoscopic optic disc assessment and the Humphrey Field Analyser (central 24-2 threshold programme). Stringent diagnostic criteria for glaucoma, based on precise optic disc and/or visual field changes, were not possible due to the retrospective nature of the study.

The PPV for glaucoma was determined according to the reason for referral. The $\chi^{2}$ test for trend was used to compare the PPV of different referral groups. When a significant trend was present, Fisher's exact test was used to compare the PPV of combined referral groups. The Kruskal-Wallis one-way analysis of variance by ranks was used to compare interval data between different referral groups.

\section{Results}

The medical records of $98.5 \%(586 / 595)$ of patients referred to the HES during the study period were reviewed. Nine records could not be found. Eighty-six referrals for suspected glaucoma were identified, which comprised $14.7 \%$ of the total. All the referrals for suspected glaucoma had been initiated by optometrists. The median age of these patients was 67 years (range 21-93 years). There were 44 men and 42 women. Table 1 gives the HES findings of these patients. The overall PPV for glaucoma was $43 \%(35 / 82)$ and the overall PPV for any abnormality was $71 \%(58 / 82)$. No diagnosis was available for 4 patients ( 3 patients defaulted and 1 patient died before attending the HES).
Table 2. Reasons for referral with suspected glaucoma

\begin{tabular}{lc}
\hline Reason for referral & No. of patients \\
\hline Raised IOP alone & 29 \\
Raised IOP + suspicious disc & 20 \\
Field loss alone & 10 \\
Raised IOP + field loss & 9 \\
Raised IOP + suspicious disc + field loss & 7 \\
Suspicious disc alone & 5 \\
Suspicious disc + field loss & 5 \\
Suspected pigmented dispersion syndrome & 1 \\
\hline
\end{tabular}

The reasons for referral with suspected glaucoma are given in Table 2 . These could be determined directly from the optometrist's referral letter for $77 \%(66 / 86)$ of patients. For the remainder, the optometrist's findings were obtained from the general practitioner's referral letter. Details regarding the following parameters were given at referral: IOP $94 \%(81 / 86)$, optic disc $76 \%(65 / 86)$ and visual field $57 \%(49 / 86)$. The methods used to assess IOP were: Pulsair tonometer (32), applanation tonometer (10) and not recorded (39). There was no significant difference between the level of IOP $(p=0.51)$ or IOP asymmetry $(p=0.30)$ among the referral groups that included raised IOP (Table 3 ). The perimeters used for screening were: Henson perimeter (16), Bjerrum screen (7), Dicon perimeter (1), Fincham-Sutcliffe perimeter (1), Park central field perimeter (1), Humphrey perimeter (1) and not recorded (22). A suprathreshold or thresholdrelated screening strategy had been used for each of the 27 patients for whom the type of perimeter had been recorded. It was not possible to analyse the different types of visual field defect found at screening because inadequate details had been given in many referral letters.

Table 4 gives the PPV for glaucoma according to the reason for referral. None of the patients referred with isolated field loss had glaucoma. This referral group comprised $12 \%$ of the glaucoma suspect referrals. Their HES findings were no abnormality (5), tilted optic disc (3) and porencephalic cyst (1). Among the referral groups that included field loss, the PPV for glaucoma increased if corroborative abnormalities were reported by the optometrist $(p=0.002)$. Referrals with isolated field loss had a significantly lower PPV for glaucoma than referrals with field loss in association with raised IOP and/or suspicious disc ( $p=0.005$, Table 5$)$. However, among the referral groups that included raised IOP or suspicious disc, the PPV for glaucoma was not significantly different if the optometrist also reported corroborative abnormalities (Tables 4,5).

Table 3. Referrals with raised IOP: referral IOP and referral IOP asymmetry

\begin{tabular}{lcc}
\hline Reason for referral & $\begin{array}{c}\text { Mean (SD, range) referral } \\
\text { IOP (mmHg) }\end{array}$ & $\begin{array}{c}\text { Mean (SD, range) referral IOP } \\
\text { asymmetry (mmHg) }\end{array}$ \\
\hline Raised IOP alone $(n=28)$ & $27.7(3.3,22$ to 37$)$ & $4.2(3.5,0$ to 12$)$ \\
Raised IOP + suspicious disc $(n=20)$ & $28.6(5.0,22$ to 39$)$ & $5.8(3.6,0$ to 13$)$ \\
Raised IOP + field loss $(n=8)$ & $26.0(2.3,23$ to 30$)$ & $3.6(3.4,0$ to 9$)$ \\
Raised IOP + field loss + suspicious disc $(n=7)$ & $30.0(6.4,22$ to 40$)$ & $7.1(6.6,1$ to 18$)$ \\
\hline
\end{tabular}

Referral IOP is based on the eye with the higher IOP ( 5 patients excluded because no value recorded). Referral IOP asymmetry is the difference between the IOP of each eye ( 8 patients excluded because no value recorded). 
Table 4. Positive predictive value (PPV) for glaucoma, or any abnormality, according to reason for referral

\begin{tabular}{|c|c|c|c|c|}
\hline Reason for referral & \multicolumn{2}{|c|}{ PPV for glaucoma ${ }^{a}$ ( $p$ value) } & \multicolumn{2}{|c|}{ PPV for any abnormality ${ }^{b}(p$ value) } \\
\hline $\begin{array}{l}\text { Referrals with field loss } \\
\text { Field loss alone }(n=9) \\
\text { Field loss + suspicious disc }(n=5) \\
\text { Field loss + raised IOP }(n=8) \\
\text { Field loss + raised IOP + suspicious disc }(n=7)\end{array}$ & $\begin{array}{l}\text { Nil } \\
40 \% \\
63 \% \\
71 \%\end{array}$ & $(p=0.002)$ & $\begin{array}{l}44 \% \\
40 \% \\
88 \% \\
86 \%\end{array}$ & $(p=0.03)$ \\
\hline $\begin{array}{l}\text { Referrals with suspicious disc } \\
\text { Suspicious disc alone }(n=4) \\
\text { Suspicious disc + field loss }(n=5) \\
\text { Suspicious disc + raised IOP }(n=20) \\
\text { Suspicious disc + raised IOP + field loss }(n=7)\end{array}$ & $\begin{array}{l}25 \% \\
40 \% \\
50 \% \\
71 \%\end{array}$ & $(p=0.12)$ & $\begin{array}{l}25 \% \\
40 \% \\
75 \% \\
86 \%\end{array}$ & $(p=0.01)$ \\
\hline $\begin{array}{l}\text { Referrals with raised IOP } \\
\text { Raised IOP alone }(n=28) \\
\text { Raised IOP + suspicious disc }(n=20) \\
\text { Raised IOP + field loss }(n=8) \\
\text { Raised IOP + field loss + suspicious disc }(n=7)\end{array}$ & $\begin{array}{l}43 \% \\
50 \% \\
63 \% \\
71 \% \\
\end{array}$ & $(p=0.13)$ & $\begin{array}{l}79 \% \\
75 \% \\
88 \% \\
86 \% \\
\end{array}$ & $(p=0.58)$ \\
\hline
\end{tabular}

Table excludes 1 patient referred with suspected pigment dispersion syndrome (normal IOP, optic discs and visual field) that was confirmed by the HES. Table also excludes the 4 patients with an unknown diagnosis. Their reasons for referral were: field loss alone (1), field loss + raised IOP (1), suspicious disc alone (1) and raised IOP alone (1).

${ }^{a}$ Includes normal tension glaucoma.

${ }^{b}$ The positive HES findings, grouped according to reason for referral, were as follows. Raised IOP alone: glaucoma (12), ocular hypertension (10). Raised IOP + suspicious disc: glaucoma (10), ocular hypertension (4), tilted optic disc (1). Field loss alone: tilted optic disc (3), porencephalic cyst (1). Raised IOP + field loss: glaucoma (5), ocular hypertension (2). Raised IOP + suspicious disc + field loss: glaucoma (5), myopic macular degeneration (1). Suspicious disc alone: glaucoma (1). Suspicious disc + field loss: normal tension glaucoma

Table 4 also gives the PPV for any abnormality being confirmed by the HES. This increased for both field loss $(p=0.03)$ and suspicious disc $(p=0.01)$ as other abnormalities were reported by the optometrist, but comparison of combined referral groups did not demonstrate a statistically significant difference (Table 5). The PPV for any abnormality was, however, significantly greater if raised IOP was a reason for referral compared with referrals for field loss and/or suspicious disc without associated raised IOP (79\% vs 39\%, $p=0.003$ ). There was no significant difference in the PPV for any abnormality between referrals with isolated field loss and referrals with isolated suspicious discs ( $44 \%$ vs $25 \%, p=0.98$ ).

\section{Discussion}

The introduction of perimetry for glaucoma screening represents an important advance. Ophthalmoscopy has poor validity as a screening test for glaucoma, especially in early cases. ${ }^{11,12}$ Tonometry is a more effective screening test ${ }^{13}$ but is limited by the absence of a single cut-off IOP that achieves a reasonable combination of sensitivity and specificity. ${ }^{12}$ While visual field testing has similar limitations, it can achieve greater efficacy as a screening test for glaucoma. ${ }^{10}$ Optimal validity for glaucoma screening is, however, currently achieved by these three screening tests in combination. ${ }^{6,14}$ Perimetry can improve the accuracy of referrals for suspected glaucoma by identifying which individuals with raised IOP or suspicious discs have glaucoma. ${ }^{15,16}$ In addition, visual field testing should increase the overall glaucoma detection rate by identifying cases that are missed by screening with ophthalmoscopy and tonometry. ${ }^{13,17}$

Normal tension glaucoma probably accounts for around $40 \%$ of all glaucoma cases in the population. ${ }^{18}$

Perimetry currently achieves acceptable validity as a screening test for glaucoma only under certain conditions. Firstly, the perimeter employed must have high sensitivity and specificity for detecting glaucomatous field loss (such as the Henson field screener ${ }^{7,8}$ ). Secondly, the prevalence of glaucoma in the screened population must be sufficient for perimetry to achieve an acceptable PPV. The PPV of a screening test, with a given sensitivity and specificity, depends on the prevalence of the target condition in the screened

Table 5. Positive predictive value (PPV) for glaucoma or any abnormality: single versus multiple reasons for referral

\begin{tabular}{|c|c|c|}
\hline Reason for referral & PPV for glaucoma ${ }^{a}$ ( $p$ value) & PPV for any abnormality ( $p$ value) \\
\hline $\begin{array}{l}\text { Referrals with field loss } \\
\text { Field loss alone } \\
\text { Field loss }+ \text { \{raised IOP and / or suspicious disc }\}\end{array}$ & $\left.\begin{array}{l}\text { Nil } \\
60 \%\end{array}\right\}$ & $(p=0.24)$ \\
\hline $\begin{array}{l}\text { Referrals with suspicious disc } \\
\text { Suspicious disc alone } \\
\text { Suspicious disc + } \text { raised IOP and/or field loss }\}\end{array}$ & $\left.\begin{array}{l}25 \% \\
53 \%\end{array}\right\} \quad(p=0.60)$ & $\begin{array}{l}25 \% \\
72 \%\end{array}$ \\
\hline $\begin{array}{l}\text { Referrals with raised IOP } \\
\text { Raised IOP alone } \\
\text { Raised IOP + \{Field loss and/or suspicious disc }\}\end{array}$ & $\left.\begin{array}{l}43 \% \\
57 \%\end{array}\right\}$ & $\begin{array}{l}79 \% \\
80 \%\end{array}$ \\
\hline
\end{tabular}

Raised IOP + \{Field loss and/or suspicious disc $\}$

${ }^{a}$ Includes normal tension glaucoma. 
population. Current methods of perimetry can achieve an acceptable PPV with selective screening of individuals at increased risk of glaucoma. ${ }^{9}$ Risk factors for glaucoma include age $>40$ years, IOP $>21 \mathrm{mmHg}$, suspicious optic discs, positive family history, diabetes, high myopia and Afro-Caribbean origin. ${ }^{6}$ Non-selective visual field screening will have a greatly reduced PPV. ${ }^{9,10}$ Thirdly, abnormal perimetry (in the absence of other features of glaucoma) should generally be repeated to confirm genuine field loss before referral. ${ }^{6}$ Perimetry is associated with numerous false positive results on initial testing that decrease with learning experience. ${ }^{10,19,20}$ Perimetric artefacts may also arise from incorrect refractive correction, incorrectly positioned corrective lenses, lid position, pupil size, media opacities and subject. fatigue.

This study assessed the PPV of visual field testing as currently practised by optometrists. Isolated field loss accounted for $12 \%$ of glaucoma suspect referrals but no cases of glaucoma detection. These subjects were either false positive referrals or had field defects of nonglaucomatous aetiology (tilted optic disc and porencephalic cyst). Inappropriate visual field testing was responsible for 4 of the 5 false positive referrals: 1 subject underwent non-selective screening (no risk factors for glaucoma) and 3 subjects were referred without repeat perimetry to confirm genuine field loss. Referrals with isolated field loss had a significantly lower PPV for glaucoma than other referral groups.

Furthermore, the presence of abnormal perimetry among referrals with raised IOP or suspicious discs did not significantly improve the PPV for glaucoma. This study could not, however, assess whether perimetry had improved glaucoma detection by reducing the false negative rate of screening by ophthalmoscopy and tonometry. Nevertheless, relatively few cases of normal tension glaucoma were detected in this study $(6 \%$ of glaucoma cases detected), suggesting that glaucoma screening still has an appreciable false negative rate.

There is no doubt that current methods of glaucoma screening need to be improved: about half the glaucoma cases in the population remain undetected ${ }^{15,19}$ and $20 \%$ of newly diagnosed cases already have advanced field loss. ${ }^{3}$ Optometrists are therefore increasingly performing perimetry to improve their glaucoma detection rate. ${ }^{21}$ This is reflected by an increasing proportion of glaucoma suspects referred with isolated field loss: $12 \%$ in this study compared with around $1 \%$ in two recent surveys. ${ }^{3,16}$ However, the findings of this study suggest that current visual field testing by optometrists is leading to unnecessary referrals to the HES with no detectable improvement in accuracy of glaucoma suspect referrals. A larger survey is required to determine the general applicability of this finding. Referrals for suspected glaucoma already constitute a significant proportion (around $15 \%{ }^{1}$ ) of all new referrals to the HES, of which approximately one-third are false positive referrals. ${ }^{3,16,22}$ It is important that the introduction of visual field testing by optometrists leads to improved glaucoma detection without an unacceptable increase in false positive referrals.

\section{References}

1. Harrison RJ, Wild JM, Hobley AJ. Referral patterns to an ophthalmic outpatient clinic by general practitioners and ophthalmic opticians and the role of these professionals in screening for ocular disease. BMJ 1988;297:1162-7.

2. Brittain GPH, Austin DJ, Kelly SP. A prospective study to determine sources and diagnostic accuracy of glaucoma referrals. Health Trends 1988;20:43-4.

3. Sheldrick JH, Ng C, Austin DJ, Rosenthal AR. An analysis of referral routes and diagnostic accuracy in cases of suspected glaucoma. Ophthalmic Epidemiol 1994;1:31-9.

4. Strong NP. How optometrists screen for glaucoma: a survey. Ophthalmic Physiol Opt 1992;12:3-7.

5. Tuck MW, Crick RP. Screening for glaucoma: the time taken by primary examiners to conduct visual field tests in practice. Ophthalmic Physiol Opt 1994;14:351-4.

6. Crick RP, Tuck MW. How can we improve the detection of glaucoma? BMJ 1995;310:546-7.

7. Henson DB, Bryson H. Clinical results with the HensonHamblin CFS2000. Doc Ophthalmol Proc Ser 1987;49:233-8.

8. Sponsel WE, Ritch R, Stamper R, Higginbotham EJ, Anderson DR, Wilson MR, Zimmerman TJ. Prevent Blindness America visual field screening study. Am J Ophthalmol 1995;120:699-708.

9. Vernon SA, Henry DJ, Jones SJ. Calculating the predictive power of the Henson field screener in a population at risk of glaucomatous field loss. Br J Ophthalmol 1990;74:220-2.

10. Katz J, Tielsch JM, Quigley HA, Javitt J, Witt K, Sommer A. Automated suprathreshold screening for glaucoma: The Baltimore Eye Survey. Invest Ophthalmol Vis Sci 1993;34:3271-7.

11. Wood CM, Bosanquet RC. Limitations of direct ophthalmoscopy in screening for glaucoma. BMJ 1987;294:1587-8.

12. Tielsch JM, Katz J, Singh K, Quigley HA, Gottsch JD, Javitt J, Sommer A. A population-based evaluation of glaucoma screening: The Baltimore Eye Survey. Am J Epidemiol 1991;134:1102-10.

13. Tuck MW, Crick RP. Relative effectiveness of different modes of glaucoma screening in optometric practice. Ophthalmic Physiol Opt 1993;13:227-32.

14. Harper R, Reeves B. Screening for primary open-angle glaucoma: a review. Ophthalmic Physiol Opt 1995;15(Suppl 2):S27-S34.

15. Vernon SA, Henry DJ, Cater L, Jones SJ. Screening for glaucoma in the community by non-ophthalmologically trained staff using semi-automated equipment. Eye 1990;4:89-97.

16. Tuck MW, Crick RP. Efficiency of referral for suspected glaucoma. BMJ 1991;302:998-1000.

17. Tuck M, Crick R. Optometrists' referral criteria for suspected glaucoma. Health Trends 1992;24:153-7.

18. Sommer A. Epidemiology as it relates to screening for glaucoma. Surv Ophthalmol 1989;33(Suppl):441-2.

19. Sheldrick JH, Sharp AJH. Glaucoma screening clinic in general practice: prevalence of occult disease, and resource implications. Br J Gen Pract 1994;44:561-5.

20. Dielemans I, Vingerling JR, Wolfs RCW, Hofman A, Grobbee DE, de Jong PTVM. The prevalence of primary open-angle glaucoma in a population-based study in the Netherlands. Ophthalmology 1994;101:1851-5.

21. Tuck MW, Crick RP. Use of visual field tests in glaucoma detection by optometrists in England and Wales. Ophthalmic Physiol Opt 1994;14:227-31.

22. Tuck MW. Referrals for suspected glaucoma: an International Glaucoma Association survey. Ophthalmic Physiol Opt 1991;11:22-6. 